\title{
Design and Fabrication of Delta Robot with Voice Control
}

\author{
G.S. Bala Maga Ganapathi*, S. Ravi Krishnamoorthy** \\ * M. Tech-Robotic, SRM University, India \\ ** School of Mechanical Engineering, SRM University, India
}

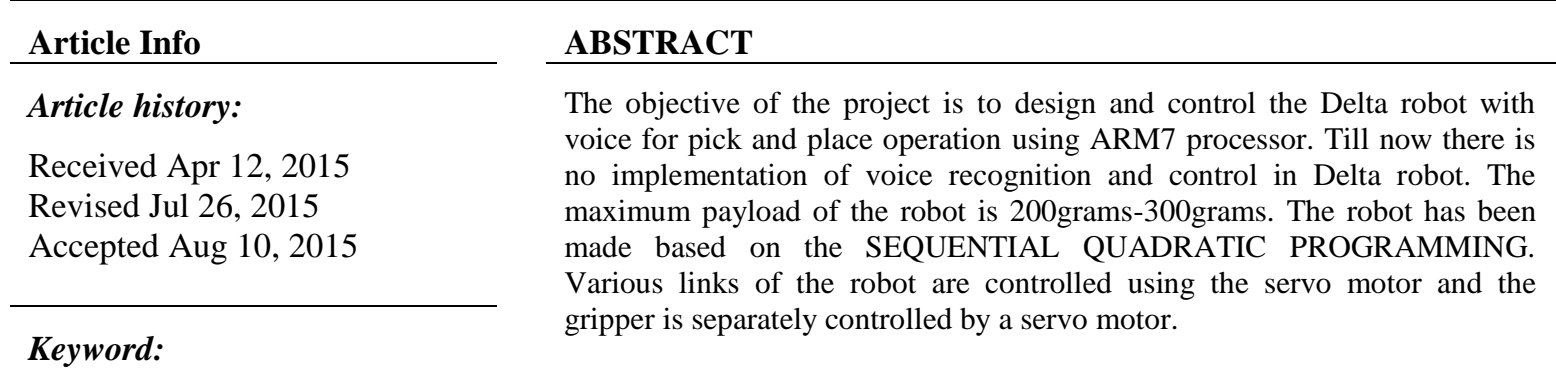

ARM7 Processor

Keil C platform

Proximal and distal links

Quadratic-Programming

Sequential

Servomotors

Copyright $\odot 2015$ Institute of Advanced Engineering and Science. All rights reserved.

Corresponding Author:

G. S. Bala Maga Ganapathi,

M. Tech-Robotic, M.Tech-Robotic, SRM University,

Chennai, India

Email: bmgmechatronics04@gmail.com

\section{INTRODUCTION}

In my project a model for the delta robot, the delta robot has arm links in which servo gripper is mounted at the end of it. Servo gripper is used for gripping action. The servo motor arrangement at the base is made to move gripper arm to four locations for pick and place purpose. Three servo motors which are placed at the joints of the each link which tend to move gripper on front, back, right and left directions. The can be picked up and placed object where required to place.

Three servo motors are used in the robot, among the three motors each distal joint has one motor. These distal joints are connected with the parallelogram joints and in turn three parallelogram joints are connected with three distal joints. Thus the three parallelogram joint are connected with the lower base plate of the robot. Then the servo controlled gripper called servo gripper is fixed with the lower base plate. This gripper is used for the pick and place operations.

The delta robot servo motors are programmed using the ARM7 microcontroller and they integrated using the servo motor driver circuit. Each servo motor is given with some pulses to make the joints actuation and based on the input pulse given to the servo motor the joints will actuate. The microcontroller have been programmed in such a way that the joint should not collide with each other. It is programmed using the Keil $\mathrm{C}$ platform. The basic Embedded C program is used to move the joints and links of the robot. It has been incorporated in the microcontroller using PHILIPS incorporator. 


\section{DESIGN AND FABRICATION}

\subsection{Methodology}

The approach towards the making of the robot has been split into different steps which start from designing, modeling, fabrication and controlling of the robot. Figure 1, depicts the steps of the methodology of work. First step is identifying the problem. The second step is developing the concept to overcome the problem which has been identified in the first step. Once the concept is developed for the identified problem, the different possibilities of the concept is sketched out and studied. The optimum solution for the concept is arrived after the studies. The various calculations are done such as kinematic parameters. Then analysis of the complex model in order to identify fundamental properties had been carried out. Then an algorithm is to be developed for the actuation of the delta robot with 3DOF using the voice command recognition and execution. Finally, the controller is interfaced with the robot for verifying the algorithm in multiple trials.

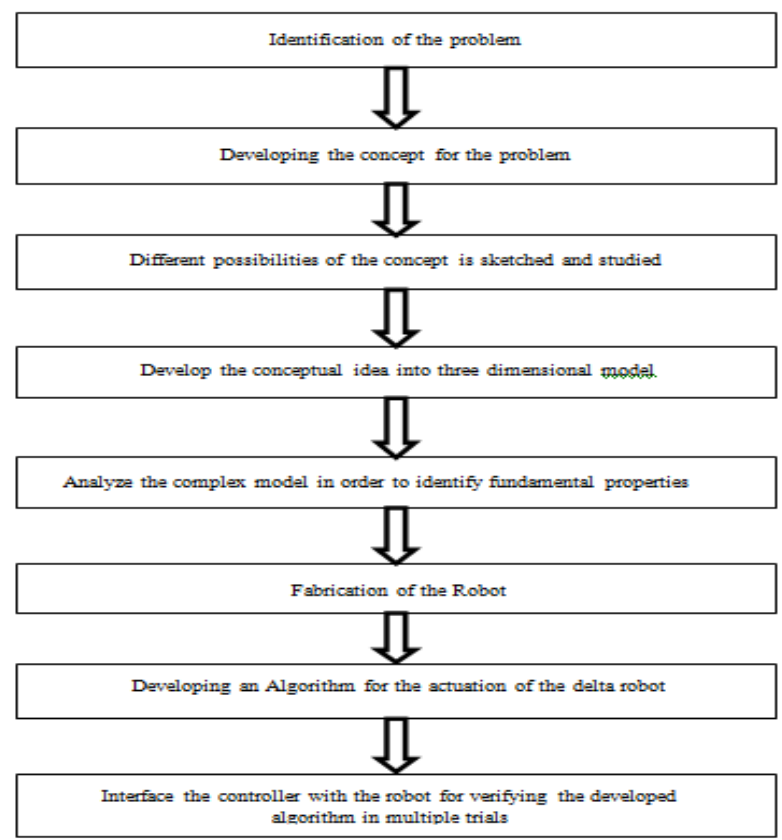

Figure 1. Flow Chart of Methodology

\subsection{Kinematics of Robot}

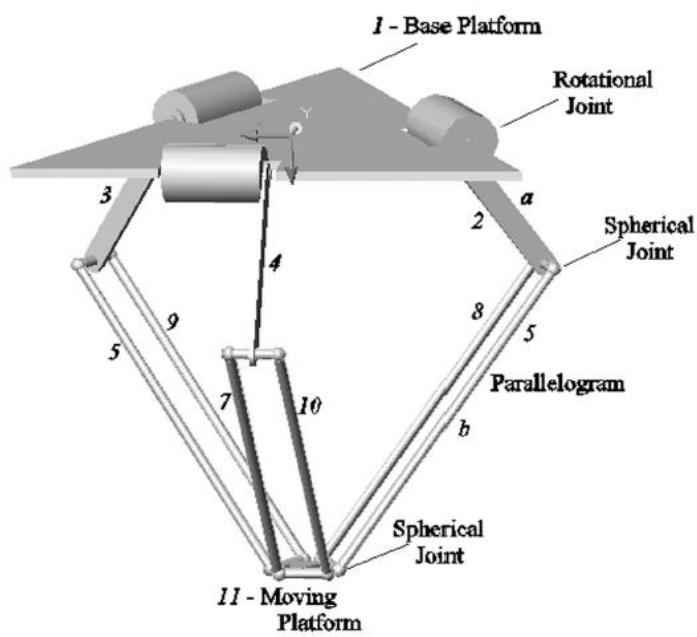

Figure 2. Kinematics of Robot

The kinematics applies geometry to the study of the movement of multi-degree of freedom kinematic chains that form the structure of robotic systems. The emphasis on geometry means that 
the links of the robot are modeled as rigid bodies and its joints are assumed to provide pure rotation or translation. Robot kinematics studies the relationship between the dimensions and connectivity of kinematic chains and the position, velocity and acceleration of each of the links in the robotic system, in order to plan and control movement and to compute actuator forces and torques.

The relationship between mass and inertia properties, motion, and the associated forces and torques is studied as part of robot dynamics. A fundamental tool in robot kinematics is the kinematics equations of the kinematic chains that form the robot. These non-linear equations are used to map the joint parameters to the configuration of the robot system. Kinematics equations are also used in biomechanics of the skeleton and computer animation of articulated characters. Forward kinematics uses the kinematic equations of a robot to compute the position of the end-effector from specified values for the joint parameters. ${ }^{[3]}$ The reverse process that computes the joint parameters that achieve a specified position of the end-effector is known as inverse kinematics. The dimensions of the robot and its kinematics equations define the volume of space reachable by the robot, known as its workspace.

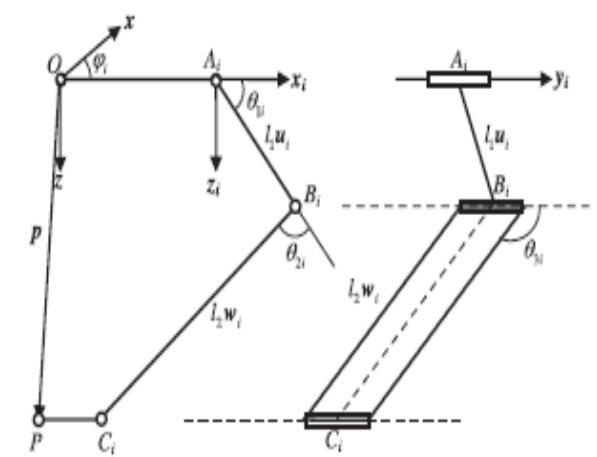

$$
\begin{aligned}
& \left(x_{d} w-x\right)^{2}+\left(y_{d} w-y\right)^{2}+\left(z_{d} w-z\right)^{2}-r_{f} w^{2}=0 \\
& \left\{\left(r_{e}^{2}-r_{f}^{2}+x_{d}^{2}-x_{c}^{2}+y_{d}^{2}-y_{c}^{2}-z_{c}^{2}\right) / 2:\left(x_{c}-x_{d}\right):\left(y_{c}-y_{d}\right): z_{c}\right\} \\
& \left|\begin{array}{cccc}
W_{\pi} & X_{\pi} & Y_{\pi} & Z_{\pi} \\
W & X & Y & Z
\end{array}\right| \Rightarrow\left\{p_{01}: p_{02}: p_{03}: p_{23}: p_{31}: p_{12}\right\} \\
& {\left[\begin{array}{cccc}
0 & p_{23} & p_{31} & p_{12} \\
-p_{23} & 0 & p_{03} & -p_{02} \\
-p_{31} & -p_{03} & 0 & p_{01} \\
-p_{12} & p_{02} & -p_{01} & 0
\end{array}\right]\left[\begin{array}{l}
w \\
x \\
y \\
z
\end{array}\right]=\left[\begin{array}{l}
0 \\
0 \\
0 \\
0
\end{array}\right]} \\
& {\left[\left(\frac{p_{01}}{p_{03}}\right)^{2}+\left(\frac{p_{02}}{p_{03}}\right)^{2}\right] z^{2}} \\
& -2\left[\frac{p_{01}}{p_{03}}\left(\frac{p_{31}}{p_{03}}+x_{d}\right)-\frac{p_{02}}{p_{03}}\left(\frac{p_{23}}{p_{03}}-y_{d}\right)\right] w z \\
& +\left[\left(\frac{p_{31}}{p_{03}}+x_{d}\right)^{2}+\left(\frac{p_{23}}{p_{03}}-y_{d}\right)^{2}+z_{d}^{2}-r_{f}^{2}\right] w^{2}=0 \\
& \mathbf{e}_{1}^{\prime}=\left[\begin{array}{c}
0 \\
e /(2 \sqrt{3}) \\
0
\end{array}\right], \quad \mathbf{e}_{2}^{\prime}=\left[\begin{array}{c}
-e / 4 \\
-e /(4 \sqrt{3}) \\
0
\end{array}\right], \quad \mathbf{e}_{3}^{\prime}=\left[\begin{array}{c}
e / 4 \\
-e /(4 \sqrt{3}) \\
0
\end{array}\right]
\end{aligned}
$$


The nine key points, $\mathrm{Di}, \mathrm{Ei}, \mathrm{Ci}$, are clearly visible. The centre of the smaller EE is displaced by (x, $\mathrm{y}, \mathrm{z}$ ) from origin $\mathrm{O}$ at the centre of FF. Note the design constants. e is the side length of EE, f the side of FF, re the distance $\mathrm{CiEi}$ and rf the distance $\mathrm{DiEi}$. A sphere, radius re, centred on $\mathrm{Ci}$ gives the locus of Ei. Furthermore a second constraint is imposed by the circular trajectory of Ei at radius rf from centre Di. The plane of this circle is visible as a line. The plane cuts the sphere in a small circle in the same plane. In the three auxiliary elevation views one sees this small circle inside the dotted outline of the sphere. The desired actuated R-joint angles are measured from the edge or line view of FF to DiEi as _i. The leg subscript $i$ is omitted in all the following equations. It is obvious that a joint angle _ must be computed separately for each leg.

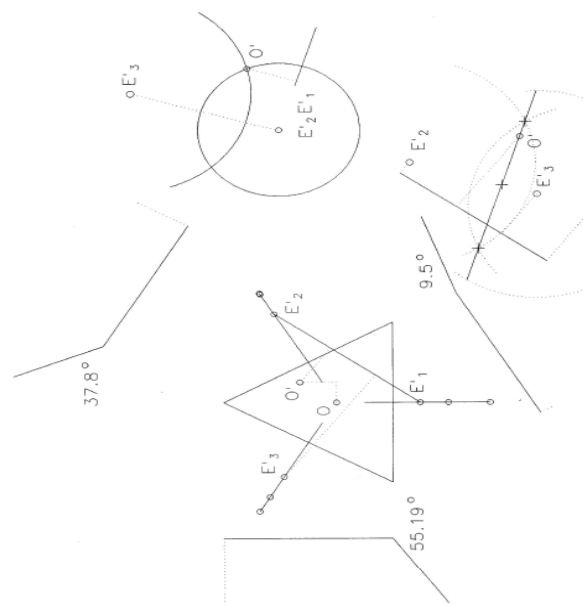

Figure 3. Key Points

These three displacement vectors e0i are seen to be and three equations, like Eq. 1, can be written. Differences between the first and second and second and third provide plane coordinates and the key line coordinates to be employed in the computationally simplest of the three sphere equations, like Eq. 3 . This is solved for the least $\mathrm{z}$-coordinate and the second and third lines of Eq. 2 produce the other two coordinates of $\mathrm{O} 0$.

\subsection{Cad Modelling of the Robot}

The Delta robot with all their components are modeled and assembled in the required structure using the SOLIDWORKS software. The 3D model of the robot is given in the below fig.

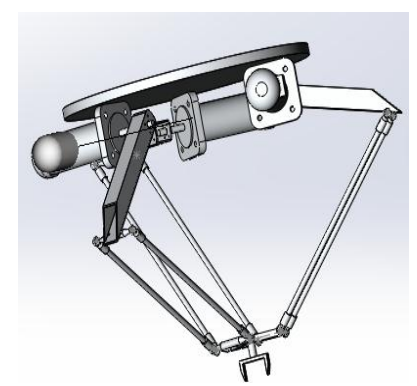

Figure 4. Modelling of the Robot

\subsection{Material Used and Calculation}

The materials selected for the manufacturing of the body is:

- Mild Steel (grade 250)

- Aluminium

Because the Acrylic material is very difficult to be get modelled as the cylindrical one. Thus it has been rejected. The cubical base frame is manufactured using the Mild Steel and the other parts like the upper 
plate, lower plate, distal link, parallelogram link and the gripper will be manufactured by using the Aluminium with less strength. The material has been selected based on the yield strength of the material (193MPa).

Weight calculation:

From the cad model the calculation of the mass have been done

Weight of the body

Weight of the base frame

Weight of the distal joint

$$
\begin{aligned}
& =4.5 \mathrm{~kg} \\
& =2.3 \mathrm{~kg}
\end{aligned}
$$

Weight of the parallelogram

$=.5 \mathrm{~kg}$

$=.6 \mathrm{~kg}$

Weight of the servo gripper

$=.35 \mathrm{~kg}$

Weight of the servo motor

$=.75 \mathrm{~kg}$

Maximum Payload of the robot

$=0.3 \mathrm{~kg}$

The total weight that the motor have to lift $=2 / 3 \mathrm{~kg}$

A motor has to lift

$$
=0.667 \mathrm{~kg}
$$

Then the torque has to be calculated using the weight of the motor that has to be lifted and it is given below. To lift heavy weight we have to go for motor with:

- high torque

- accuracy

- high speed

So, i selected the servomotor for the precision and accuracy and the torque calculation is given by

$\mathrm{F}=\mathrm{m} * \mathrm{a}$

$\mathrm{F}=67.18 * 9.81$

\section{Force $=660 \mathrm{~N}$}

Work $=$ force $*$ distance

Work $=660 * 550 \mathrm{~mm}$

\section{Work $=363 \mathrm{Nm}$}

Power $=2 * 3.14 * \mathrm{~N} * \mathrm{~T} / 60$

$\mathrm{T}=363 * 60 /(2 * 3.14 * 1500)$

\section{Torque $=2.3 \mathrm{Nm}$}

Hence a motor with torque greater than or equal to $2.3 \mathrm{Nm}$ has been selected.

\section{Motor Selection:}

Since the delta robot has been used for accurate picking and placing of the object, the perfect motor for the operations is SERVOMOTOR.

A servomotor is a rotary actuator that allows for precise control of angular position, velocity and acceleration. It consists of a suitable motor coupled to a sensor for position feedback. It also requires a relatively sophisticated controller, often a dedicated module designed specifically for use with servomotors. Servomotors are not a specific class of motor although the term servomotor is often used to refer to a motor suitable for use in a closed-loop control system.

Servo motor should be connected with some microcontroller in order to make it actuated. In the microcontroller a program will be incorporated to actuate the servomotor, in between the there is a need of motor driver circuit to make the connection of the servomotors with the microcontroller. Through the motor driver circuit the servo motor is controlled as well as drove in accordance with our need. The connection of the motor with the microcontroller can be given by:

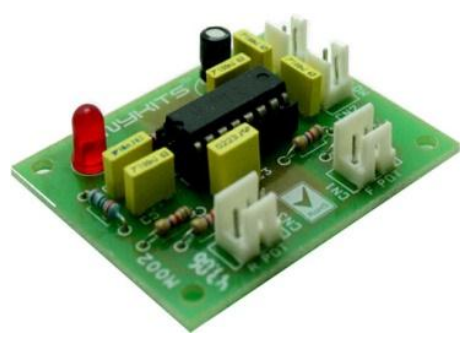

Figure 5. Servomotor 


\subsection{Servo Gripper}

The servo gripper has been used for the pick and place operation of the robot. This is selected because of the best gripping and precisely works. Gripper Assembly mates easily with two servos, one to open and close the fingers, and a second servo which acts as the gripper's "wrist". The gripper can accommodate objects up to about .9" (23 mm). Compatible with many Futaba and HiTec servo motors,
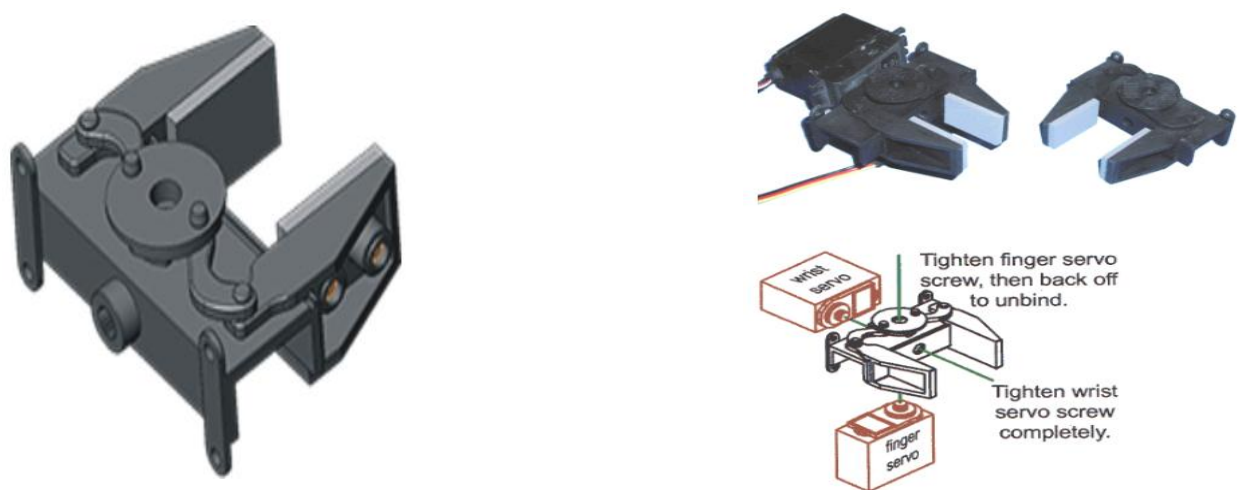

Figure 6. Servo Gripper

The servo gripper has the biggest advantage that there is no need of separate servo motor to actuate the servo gripper. It has the in-built servo motor setup with it and it makes the actuation with high accuracy for the pick and place operation. The most important feature is the accuracy as well as the controlling of the servo gripper is quite easy by the user. Servo gripper is the favourable selection of the gripper for picks and place operation.

The jaws open to 1.3" and the wrist rotates approximately 180 degrees for open and close and wrist rotate. The servos are not included.

Microcontrollers are used in automatically controlled products and devices, such as automobile engine control systems, implantable medical devices, remote controls, office machines, appliances, power tools, toys and other embedded systems. By reducing the size and cost compared to a design that uses a separate microprocessor, memory, and input/output devices, microcontrollers make it economical to digitally control even more devices and processes. Mixed signal microcontrollers are common, integrating analog components needed to control non-digital electronic systems. The microcontroller used for this project is the latest controller existing in the electronics market ARM7 MICROCONTROLLER.

\section{VOICE RECOGNITION AND CONTROL}

The voice recognition and control of the robot is the tedious part in this paper and after several trials of various ICs and came to the conclusion of using HM2007 IC to be the voice processing IC.

\subsection{Voice Control Ic-Hm2007}

The speech recognition system is a completely assembled and easy to use programmable speech recognition circuit. Programmable, in the sense that you train the words (or vocal utterances) you want the circuit to recognize. This board allows you to experiment with many facets of speech recognition technology. It has 8 bit data out which can be interfaced with any microcontroller for further development. Some of interfacing applications which can be made are controlling home appliances, robotics movements, Speech Assisted technologies, Speech to text translation, and many more. The features of the voice recognition and control can be given as below

a. Self-contained stand alone speech recognition circuit

b. User programmable

c. Up to 20 word vocabulary of duration two second each

d. Multi-lingual

e. Non-volatile memory back up with $3 \mathrm{~V}$ battery onboard. Will keep the speech recognition data in memory even after power off.

f. Easily interfaced to control external circuits \& appliances 
Table 1. The specifications of the HM2007 is given below

\begin{tabular}{lll}
\hline Parameter & Value & Note \\
\hline Input Voltage & 9 to $15 \mathrm{~V} \mathrm{DC}$ & Use a commonly available 12V 500ma DC Adapter \\
Output Data & 8 bits at 5V & Any microcontroller like 8051, PIC or AVR can be interfaced to data \\
& Logic Level & port to interpret and implement specialized applications \\
\hline
\end{tabular}

The HM 2007 is the form of Integrated Chip and it can be looks as in the below one with all the pins integrated in it.
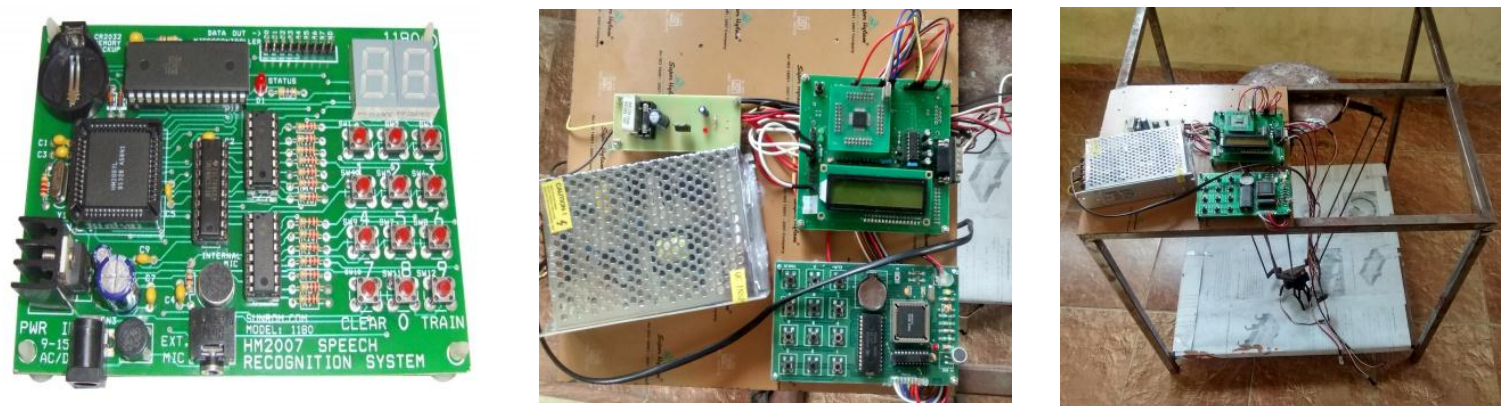

Figure 7. Integrated Chip HM2007

The has been connected with the microcontroller and has been programmed using the Keil $\mathrm{C}$ with the help of the Embedded C. The Delta robot program has been incorporated in to the microcontroller ARM7 has been done by PHILIPS incorporator software. The SNPS is connected in order to give the power required by the servomotor as well as the microcontroller to start its process up. The LCD is added along with the system to know whether the links moving towards which direction and to follow our commands for proper operation.

The Delta robot prototype has been developed and checked with the voice recognition whether it can move in the right direction or not and also the servo gripper has been controlled by using the voice commands only. Hence the delta robot can be used with voice to make its manipulation. The complete setup of the Robot is as given below.

\section{CONCLUSION}

The Delta robot prototype has been developed along with the control system. The ARM7 controller is adaptable for this process and also it is the advanced controller too. Voice recognitions and controls can be done by connecting and programming the HM-2007 IC with the microcontroller. The ultimate target of the pick and place operation has been done along with the prescised accuracy. The problem which we faced with the robot is the speed with the voice control is not up to the level of commercially available robot. In future phase it has to be enhanced to meet the speed of the commercially available pick and place delta robot.

\section{REFERENCES}

[1] Yunjiang Lou, Yongsheng Zhang, Ruining Huang, Xin Chen and Zexiang Li, "Optimization Algorithms for Kinematically Optimal Design of Parallel Manipulators", IEEE TRANSACTIONS ON AUTOMATION SCIENCE AND ENGINEERING, April $19^{\text {th }} 2014$.

[2] Yongjie Zhao "Dimensional synthesis of a three translational degrees of freedom parallel robot while considering kinematic anisotropic property" in Science Direct Robotics and Computer-Integrated Manufacturing, November $6^{\text {in }}$ 2013.

[3] M.A. Laribi, L. Romdhane and S. Zeghloul "Analysis and dimensional synthesis of the DELTA robot for a prescribed workspace" in Science Direct Mechanism and Machine Theory, November $4^{\text {th }} 2007$.

[4] Arturo Gil, Adrián Peidró, Óscar Reinoso and José María Marín "Implementation and Assessment of a Virtual Laboratory of Parallel Robots Developed for Engineering Student" in IEEE TRANSACTIONS ON EDUCATION, Vol. 57, No. 2, May 2014.

[5] Y.H. Li, Y. Ma, S.T. Liu, Z.J. Luo, J.P. Mei, T. Huang and D.G. Chetwynd "Integrated design of a 4-DOF highspeed pick-and-place parallel robot" in Science Direct CIRP Annals - Manufacturing Technology, 2014.

[6] Qiang Zeng, KornelF. Ehmann and JianCao "Tri-pyramid Robot: Design and kinematic analysis of a 3-DOF translational parallel manipulator" in Science Direct Robotics and Computer-Integrated Manufacturing, 2014. 
[7] Tian Huang, Songtao Liu, Jiangping Mei and Derek G. Chetwynd "Optimal design of a 2-DOF pick-and-place parallel robot using dynamic performance indices and angular constraints" in Science Direct Mechanism and Machine Theory, 2013.

[8] Ridha Kelaiaia, Olivier Company and Abdelouahab Zaatri "Multi objective optimization of a linear Delta parallel robot" in Science Direct Mechanism and Machine Theory, 2012.

[9] Stanislas Oger and Georges Linarès "Web-based possibilistic language models for automaticspeech recognition" in Science Direct Computer Speech and Language, 2014.

[10] SabatoMarcoSiniscalchi, TorbjørnSvendsen and Chin-HuiLee "An artificial neural network approach to automatic speech processing” in Science Direct Neurocomputing, 2014.

[11] Andreas Stolcke, Barry Chen, Horacio Franco, Venkata Ramana Rao Gadde, Martin Graciarena, Mei-Yuh Hwang, Katrin Kirchhoff, Arindam Mandal, Nelson Morgan, Xin Lei, Tim Ng, Mari Ostendorf, Kemal Sönmez, Anand Venkataraman, Dimitra Vergyri, Wen Wang, Jing Zheng and Qifeng Zhu "Recent Innovations in Speech-to-Text Transcription at SRI-ICSI-UW" in IEEE TRANSACTIONS ON AUDIO, SPEECH, AND LANGUAGE PROCESSING, Vol. 14, No. 5, September 2006.

[12] Hai-Son Le, Ilya Oparin, Alexandre Allauzen, Jean-Luc Gauvain and François Yvon "Structured Output Layer Neural Network Language Models for Speech Recognition" in IEEE TRANSACTIONS ON AUDIO, SPEECH, AND LANGUAGE PROCESSING, Vol. 21, No. 1, January 2013.

[13] Vikrant Singh Tomar and Richard C Rose "A Family of Discriminative Manifold Learning Algorithms and Their Application to Speech Recognition" in IEEE/ACM TRANSACTIONS ON AUDIO, SPEECH, AND LANGUAGE PROCESSING, Vol. 22, No. 1, January 2014.

[14] Langzhou Chen, Mark J. F. Gales, Norbert Braunschweiler, Masami Akamine and Kate Knill "Integrated Expression Prediction and Speech Synthesis From Text" in IEEE JOURNAL OF SELECTED TOPICS IN SIGNAL PROCESSING, Vol. 8, No. 2, April 2014.

[15] Haojin Yang and Christoph Meinel "Content Based Lecture Video Retrieval Using Speech and Video Text Information" in IEEE TRANSACTIONS ON LEARNING TECHNOLOGIES, Vol. 7, No. 2, April-June 2014. 